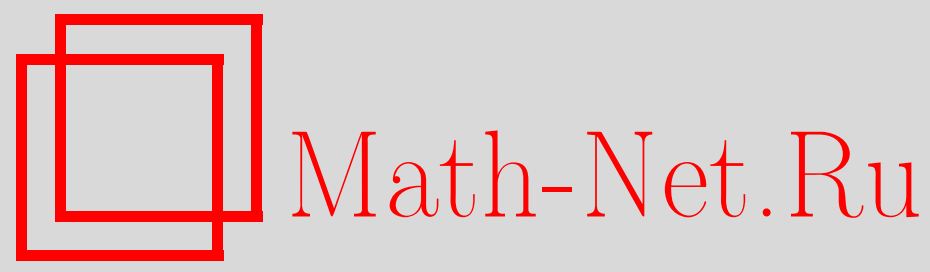

В. А. Антонов, И. А. Тюрина, А. П. Ческидов, О конечных группах с ограничениями на централизаторы, Матем. заметки, 2002, том 71, выпуск 4, 483-495

DOI: https://doi.org/10.4213/mzm360

Использование Общероссийского математического портала Math-Net.Ru подразумевает, что вы прочитали и согласны с пользовательским соглашением http://www . mathnet.ru/rus/agreement

Параметры загрузки:

IP: 34.227 .88 .159

26 апреля 2023 г., 18:00:47

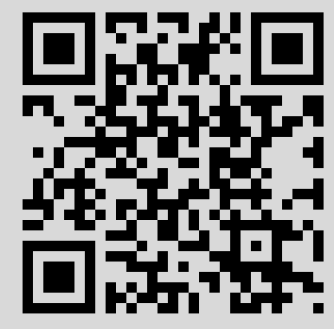




\title{
О КОНЕЧНЫХ ГРУППАХ \\ С ОГРАНИЧЕНИЯМИ НА ЦЕНТРАЛИЗАТОРЫ
}

\section{В. А. Антонов, И. А. Тюрина, А. П. Ческидов}

\begin{abstract}
Через $w(n)$ условимся обозначать количество множителей в представлении натурального числа $n$ в виде произведения простых чисел. Если $H$ - подгруппа конечной группы $G$, то $w(H)=w(|H|)$ и $v(G)=\max \{w(C(g)) \mid g \in G \backslash Z(G)\}$. В данной работе приводится полное описание групп с нетривиальным центром с условием $v(G)=4$.

Библиография: 10 названий.
\end{abstract}

Через $w(n)$ условимся обозначать количество множителей в представлении натурального числа $n$ в виде произведения простых чисел. Если $H$ - подгруппа конечной группы $G$, то $w(H)=w(|H|)$ и

$$
v(G)=\max \{w(C(g)) \mid g \in G \backslash Z(G)\} .
$$

В работах [1] и [2] описаны конечные неабелевы групшы с условиями $v(G)=2$ и $v(G)=3$ соответственно. В [3] исследованы конечные групшы без центра с условием $v(G)=4$. В предлагаемой работе приводится описание груп с нетривиальным центром, для которых $v(G)=4$. Это завершает полное описание конечных неабелевых групा с таким условием.

1. m-группы. Следуя [4], $m$-группой будем назьвать неабелеву группу, все собственные централизаторы в которой абелевы.

ЛЕмма 1.1. Пусть $G$ - конечная неабелева $p$-группа с условием $v(G)=n$. Если $|Z(G)|=p^{m}$, mo $p^{n+1} \leqslant|G| \leqslant p^{n+m}$.

ДокаЗАтЕЛЬСтво. Пусть $x \in Z_{2}(G) \backslash Z(G), f: g \rightarrow[g, x]$. Тогда из $|G|=|\operatorname{Ker} f|$. $|\operatorname{Im} f|$ и того, что Ker $f=C(x), \operatorname{a} \operatorname{Im} f \leqslant Z(G)$, получаем $|G| \leqslant p^{n} \cdot p^{m}=p^{n+m}$.

Заметим, что из $Z(G)<C(x)$ следует, что $m<n$. Поэтому $|G| \leqslant p^{2 n-1}$. В частности, для $n=4$ получаем $p^{5} \leqslant|G| \leqslant p^{7}$. При этом если $|Z(G)|=p$, то $|G|=p^{5}$; если $|Z(G)|=p^{2}$, то $|G| \leqslant p^{6}$, и если $|Z(G)|=p^{3}$, то $|G| \leqslant p^{7}$. И обратно, если $|G|=p^{7}$, то $|Z(G)|=p^{3}$, если $|G|=p^{6}$, то $p^{3} \geqslant|Z(G)| \geqslant p^{2}$, и если $|G|=p^{5}$, то $p^{3} \geqslant|Z(G)| \geqslant p$.

В дальнейшем будем писать $Z$ вместо $Z(G)$ и $Z_{2}$ вместо $Z_{2}(G)$. Кроме того, $S_{p}=$ $S_{p}(G)$ - силовская $p$-подгрупша групшы $G$ и $C^{2}(x)=C(C(x))$.

При доказательстве всех теорем мы ограничимся доказательством необходимости. Достаточность везде проверяется непосредственно.

Так как в нильпотентной $m$-группе только одна силовская $p$-подгруппа неабелева, то в силу [1], [2] для описания нильпотентных $m$-групп нам достаточно ограничиться $p$-группами. 
Теорема 1.2. Конечная p-группа $G$ в том и только том случае является $m$-группой с условием $v(G)=4$, когда выполняется один из следующих случаев:

1) $|G|=p^{5}$ и $G$ изоклинна одной из следующих әрупп:

$$
\begin{aligned}
& G_{1}=\langle a\rangle \lambda\langle b\rangle, a^{p^{2}}=b^{p}=1,[a, b]=a^{p} \\
& G_{2}=\langle a\rangle \lambda\langle b\rangle, a^{p^{3}}=b^{p}=1,[a, b]=a^{p} ; \\
& G_{3}=\langle a\rangle \lambda\langle b\rangle, a^{p^{4}}=b^{p}=1,[a, b]=a^{p} ; \\
& G_{4}=\left(\left\langle a_{1}\right\rangle \times\left\langle a_{2}\right\rangle\right) \lambda\langle b\rangle, a_{i}^{p^{2}}=b^{p}=1,\left[a_{i}, b\right]=a_{i}^{p}, i=1,2 ;
\end{aligned}
$$

2) $|G|=p^{6}$ и $G$ изоклинна одной из следующих групп:

$G_{5}=\left(\left(\left\langle z_{1}\right\rangle \times\left\langle z_{2}\right\rangle \times\left\langle z_{3}\right\rangle \times\langle a\rangle\right) \lambda\left\langle b_{1}\right\rangle\right) \lambda\left\langle b_{2}\right\rangle, z_{i}^{p}=a^{p}=b_{j}^{p}=1$ для $i=1,2,3 ;$ $j=1,2 ;\left[z_{i}, b_{j}\right]=1,\left[a, b_{j}\right]=z_{j},\left[b_{1}, b_{2}\right]=z_{3}$;

$G_{6}=\left(\left(\left\langle z_{1}\right\rangle \times\left\langle z_{2}\right\rangle \times\left\langle z_{3}\right\rangle \times\langle a\rangle\right) \lambda\left\langle b_{1}\right\rangle\right) \lambda\left\langle b_{2}\right\rangle, z_{i}^{p}=a^{p}=b_{j}^{p}=1$ для $i=1,2,3$, $j=1,2 ;\left[z_{i}, b_{j}\right]=1,\left[a, b_{j}\right]=z_{j},\left[b_{1}, b_{2}\right]=a, p>2$;

$G_{7}=\left(\left\langle z_{1}\right\rangle \times\left\langle z_{2}\right\rangle \times\left\langle a_{1}\right\rangle \times\left\langle a_{2}\right\rangle\right) \lambda\left(\left\langle b_{1}\right\rangle \times\left\langle b_{2}\right\rangle\right), z_{i}^{p}=a_{i}^{p}=b_{i}^{p}=1$ для $i=1,2$; $\left[a_{1}, b_{1}\right]=z_{1},\left[a_{1}, b_{2}\right]=\left[a_{2}, b_{1}\right]=z_{2},\left[a_{2}, b_{2}\right]=z_{1}^{\alpha_{1}} z_{2}^{\alpha_{2}}$ и уравнение $x^{2}+\alpha_{2} x-\alpha_{1}$ $=0$ не имеет решений в $G F(p)$;

$G_{8}=\left(\left(\left\langle z_{1}\right\rangle \times\left\langle z_{2}\right\rangle \times\left\langle a_{1}\right\rangle \times\left\langle a_{2}\right\rangle\right) \lambda\left\langle b_{1}\right\rangle\right) \lambda\left\langle b_{2}\right\rangle, z_{i}^{p}=a_{i}^{p}=b_{i}^{p}=1$ для $i=1,2$; $\left[b_{1}, b_{2}\right]=a_{1},\left[a_{1}, b_{i}\right]=z_{i},\left[a_{2}, b_{1}\right]=z_{1}^{\alpha_{1}} z_{2}^{\beta_{1}},\left[a_{2}, b_{2}\right]=z_{1}^{\alpha_{1}} z_{2}^{\beta_{2}}, p \neq 2$ и уравнение $x^{2}-\left(\alpha_{1}+\beta_{2}\right) x-\alpha_{2} \beta_{1}=0$ не имеет решений в $G F(p)$.

ДокАЗАТЕЛьство. В силу леммы $1.1|G| \in\left\{p^{5}, p^{6}, p^{7}\right\}$.

Пусть $|G|=p^{5}, a \in Z_{2} \backslash Z$. Тогда $|C(a)|=p^{4}$, т.е. $G$ обладает абелевой максимальной подгруппой. Холл [5] показал, что если $G$ и $H$ - две $p$-группы с абелевой максимальной подгруппой, то $G$ изоклинна $H$ тогда и только тогда, когда $\left|\gamma_{i}(G) / \gamma_{i+1}(G)\right|=\mid \gamma_{i}(H) /$ $\gamma_{i+1}(H) \mid$ для $i \geqslant 2$. При этом $\left|\gamma_{i}(G) / \gamma_{i+1}(G)\right| \geqslant\left|\gamma_{i+1}(G) / \gamma_{i+2}(G)\right|$. Пусть $c-$ ступень нильпотентности групшы $G$ и $\left(\alpha_{1}, \alpha_{2}, \ldots, \alpha_{c-1}\right)$ - набор чисел со свойством $\mid \gamma_{i}(G) /$ $\gamma_{i+1}(G) \mid=p^{\alpha_{i-1}}$. Несложно заметить, что наборы $(3)$ и $(2,1)$ невозможны. Остаются случаи $(1),(1,1),(1,1,1)$ и $(2)$, т.е. $G$ изоклинна одной из групп $G_{1}, G_{2}, G_{3}, G_{4}$.

Пусть теперь $|G|=p^{6}$. В этом случае в $G$ нет абелевых максимальных подруп. Если $a \in Z_{2} \backslash Z$, то $C(a)$ - инвариантный в $G$ централизатор. В силу теоремы 2.5 из [6] можно считать, что $C(a)$ - группа экспоненты $p$ и $G$ порождается элементами порядка $p$.

Предположим сначала, что $|Z|=p^{3}$. Тогда можно считать, что

$$
G=\left(\left(\left\langle z_{1}\right\rangle \times\left\langle z_{2}\right\rangle \times\left\langle z_{3}\right\rangle \times\langle a\rangle\right) \lambda\left\langle b_{1}\right\rangle\right) \lambda\left\langle b_{2}\right\rangle,
$$

$\left[a, b_{i}\right]=z_{i}$. Если $\left[b_{1}, b_{2}\right]=z_{1}^{\alpha} z_{2}^{\beta}$, то $\left[a^{-\beta} b_{1}, a^{\alpha} b_{2}\right]=1$, что противоречит тому, что $v(G)=4$. Поэтому можно считать, что либо $\left[b_{1}, b_{2}\right]=z_{3}$, либо $\left[b_{1}, b_{2}\right]=a$. Так как собственные централизаторы группы $G$ индуцируют расщепление фактор-групшы $G / Z$, то $G / Z$ - групша экспоненты $p$. Поэтому во втором случае $p \neq 2$, и мы получаем одну из групп $G_{5}$ или $G_{6}$.

Пусть теперь $|Z|=p^{2}$, т.е.

$$
G=\left(\left(\left\langle z_{1}\right\rangle \times\left\langle z_{2}\right\rangle \times\left\langle a_{1}\right\rangle \times\left\langle a_{2}\right\rangle\right) \lambda\left\langle b_{1}\right\rangle\right) \lambda\left\langle b_{2}\right\rangle .
$$

Можно считать, что $\left[a_{1}, b_{i}\right]=z_{i}$. Если для любого $x \in G \backslash Z(G)$ выполняется равенство $|C(x)|=p^{4}$, то $G$ - группа первого типа из [7] и, следовательно, изоклинна $G_{7}$. Поэтому будем считать, что $C\left(b_{1}\right)=Z\left\langle b_{1}\right\rangle$. Если бы ступень нильпотентности группы $G$ была равна 2 , то из зависимости элементов $\left[a_{1}, b_{1}\right],\left[a_{2}, b_{1}\right],\left[b_{2}, b_{1}\right]$ мы бы получили 
противоречие с тем, что $C\left(b_{1}\right)=Z\left\langle b_{1}\right\rangle$. Следовательно, ступень $G$ больше двух. Тогда, как и выше, $p>2$.

Так как $Z_{2} \leqslant C\left(G^{\prime}\right)<G$, то $Z_{2}$ абелева, т.е. $Z_{2} \leqslant C\left(a_{1}\right)$. Если $\left[a_{2}, b_{1}\right] \in a_{1}^{\alpha} Z$ и $\left[a_{2}, b_{2}\right] \in a_{1}^{\beta} Z$, то $\left[a_{2}, b_{1}^{\beta} b_{2}^{-\alpha}\right] \in Z$, т.е. можно считать, что $\left[a_{2}, b_{1}\right] \in Z$. Но тогда из $\left[a_{2}, b_{1}, b_{2}\right]=\left[a_{2}, b_{2}, b_{1}\right]$ (см. доказательство леммы 3.11 из [6]) получим $\left[a_{2}, b_{2}\right] \in A \cap$ $C\left(b_{1}\right)=Z$, т.е. $a_{2} \in Z_{2}$.

Итак, $Z_{2}=C\left(a_{1}\right)$. Пусть $\left[a_{2}, b_{1}\right]=z_{1}^{\alpha_{1}} z_{2}^{\beta_{1}},\left[a_{2}, b_{2}\right]=z_{1}^{\alpha_{2}} z_{2}^{\beta_{2}}$. Так как $C(a) \cap C\left(b_{1}^{s} b_{2}^{t}\right)$ $=Z$ для $b_{1}^{s} b_{2}^{t} \neq 1$ и $a \in Z_{2} \backslash Z$, то равенство $\left[a_{1}^{x} a_{2}^{y}, b_{1}^{s} b_{2}^{t}\right]=1$ может вьполняться только при $s=t=0$ или $x=y=0$.

Из $\left[a_{1}^{x} a_{2}^{y}, b_{1}^{s} b_{2}^{t}\right]=z_{1}^{x s+\alpha_{1} y s+\alpha_{2} y t} z_{2}^{x t+\beta_{1} y s+\beta_{2} y t}$ получаем, что система

$$
\left\{\begin{array}{l}
x s+\left(\alpha_{1} s+\alpha_{2} t\right) y=0 \\
x t+\left(\beta_{1} s+\beta_{2} t\right) y=0
\end{array}\right.
$$

имеет единственное решение при любых $s$ и $t$, не равных нулю одновременно, т.е. система

$$
\left\{\begin{array}{l}
\alpha_{1} s+\alpha_{2} t=k s \\
\beta_{1} s+\beta_{2} t=k t
\end{array}\right.
$$

не имеет решений при всех $k \in G F(p)$. Следовательно, уравнение $k^{2}-\left(\alpha_{1}+\beta_{2}\right) k-\alpha_{2} \beta_{1}$ $=0$ не имеет решений и $G$ изоклинна $G_{8}$.

Наконец, предположим, что $|G|=p^{7}$. Тогда $|Z|=p^{3}$ и $|G: C(x)|=p^{3}$ для любого $x \in G \backslash Z$. По теореме 3.6 из [8] $G$ должна быть двуступенно нильпотентной. Но тогда, очевидно, $\left|G^{\prime}\right|=p^{3}$ и по теореме 3.3 из [8] $G$ изоклинна полуэкстраспециальной групше, т.е. должно вьполняться неравенство $p^{7}=|G| \geqslant|Z| \cdot|G: C(x)|^{2}=p^{9}$, что невозможно.

Теорема 1.3. Ненильпотентная группа $G$ в том и только том случае является $m$-группой с условием $v(G)=4$, когда $G$ - одна из следующих групп:

1) $G=(A \times B)\langle b\rangle, A$ и $B$ - абелевь $p$ - и $p^{\prime}$-группы соответственно, $b^{p} \in A$, $A\langle b\rangle$ и $B \lambda\langle b\rangle$ неабелевы, $w(A \times B)=4$;

2) $G / Z=A / Z \lambda B / Z$ - группа Фробениуса, подгруппы $A$ и $B$ абелевы, $\max \{w(A)$, $w(B)\}=4$

3) $G / Z=A / Z \lambda B / Z$ - әруппа Фробениуса, $B$ абелева, $A$ является $m$-группой, $A / Z$ примарна, $Z(A)=Z, \max \{v(A), w(B)\}=4$

4) $G / Z \cong S_{4}, w(Z)=2$ и если $V / Z$ - четверная группа Клейна, то $V$ неабелева;

5) $G=P S L(2,9)^{*}$ - накрывающая группа для $P S L(2,9)$;

6) $G=S L(2, q), q \in\left\{2^{4}, 5^{3}, 7^{3}, 5^{2}, p\right\}$, әде $p$ - простое число со свойством $\max \{w(p-1), w(p+1)\}=4$

7) $G=A \times S L(2, q), q \in\{4,8\}, A-$ абелева группа, $w(A)=1$ при $q=8 u$ $w(A)=2$ npu $q=4$

8) $G=S L(2, q) Z, q \in\{5,9,27, p\}$, әде $p$ - простое число со свойством $\max \{w(p-1), w(p+1)\}=3, w(Z)=3$ nри $q=5 u w(Z)=2$ в остальных случаях;

9) $G / Z \cong P G L(2, q), G^{\prime} \cong S L(2, q)$ u либо $w(Z)=2 u q=5$, либо $w(Z)=1 u$ $q \in\{9,27, p\}$, где $p$ - простое число из пункта 8$)$. 
ДоКАЗАТЕЛЬСТво. В силу [4, теоремы 5.9 и 5.12$]$ группа $G$ удовлетворяет одному из следующих свойств:

1) $G$ обладает абелевым нормальным делителем простого индекса $p$;

2) $G / Z=A / Z \lambda B / Z$ - группа $\Phi$ робениуса, $B$ - абелева группа, а группа $A$ либо абелева, либо $Z(A)=Z$ и $A / Z$ примарна;

3) $G / Z \cong S_{4}$ и если $V / Z$ - четверная группа Клейна, то $V$ неабелева;

4) $G / Z \cong P S L\left(2, p^{n}\right)$ или $P G L\left(2, p^{n}\right), p^{n}>3$, и либо $G^{\prime} \cong S L\left(2, p^{n}\right)$, либо $p^{n}=9$ и $G^{\prime} \cong P S L(2,9)^{*}$.

Учитывая, что $v(G)=4$, в случаях 1 )-3) получаем групшы типов 1$)-4)$ из формулировки теоремы. Рассмотрим случай 4$)$.

Если $G^{\prime} \cong P S L(2,9)^{*}$, то из $G \geqslant G^{\prime} Z$ и $v(G)=4$ получаем группу типа 5$)$.

Предположим, что $G / Z \cong P S L\left(2, p^{n}\right), G^{\prime} \cong S L\left(2, p^{n}\right)$. Тогда $G=G^{\prime} Z$. Если $G^{\prime}<G$, то $v\left(G^{\prime}\right) \leqslant 3$ и в силу [1], [2] мы получаем группы типов 7), 8).

Пусть теперь $G=S L\left(2, p^{n}\right)$. Так как $v(G)=4$, то $n \leqslant 4$. Если $A$ - собственный централизатор из $G$, то (см. [4, доказательство теоремы 5.9]) $A / Z$ является либо элементарной абелевой группой порядка $p^{n}$, либо циклической группой порядка $\left(p^{n} \pm 1\right) / d$, где $d=\left(p^{n}-1,2\right)$. Поэтому либо $p=2$ и $n=4$, либо $p>2$ и $n \leqslant 3$. Рассмотрим второй случай.

Если $n=1$, то должно вьполняться условие

$$
\max \{w(p-1), w(p+1)\}=4
$$

Пусть $n=2$. Из $p^{2}-1=8 \cdot\left(p^{2}-1\right) / 8$ следует, что $\left(p^{2}-1\right) / 8-$ простое число. Так как при $p \neq 3$ число $p^{2}-1$ делится на 3 , то $\left(p^{2}-1\right) / 8=3$ и $p=5$.

В случае $n=3$ предположим сначала, что $p \equiv 1(4)$. Тогда $p^{3}-1=4 \cdot(p-1) / 4$. $\left(p^{2}+p+1\right)$. Если $p \neq 5$, то $(p-1) / 4$ и $p^{2}+p+1$-простые числа. Так как $|G|$ делится на 3 , то на 3 делится число $p^{3}+1$. Из $p^{3}+1=18 \cdot(p+1) / 6 \cdot\left(p^{2}-p+1\right) / 3$ получаем $v(G)>4$. В случае $p \equiv-1(4)$ и $p>7$ аналогично получим, что $v(G)>4$.

Наконец, если $G / Z \cong P G L\left(2, p^{n}\right), G^{\prime} \cong S L\left(2, p^{n}\right), p>2$, и $A$ - собственньй централизатор, то (см. [4, доказательство теоремы 5.9]) $A / Z$ - либо элементарная абелева порядка $p^{n}$, либо циклическая группа порядка $p^{n} \pm 1$. Так как $Z>1$, то $w\left(p^{n}-1\right)$ и $w\left(p^{n}+1\right)$ не превосходят 3, и мы получаем групу типа 9$)$.

2. Группы с условием $Z_{2}>Z$. Если $G$ - непримарная нильпотентная группа с условием $v(G)=4$, то, очевидно, $G$ является $m$-группой.

ЛЕмма 2.1. Пусть $G$ - конечная неабелева $p$-группа с условием $v(G)=4$. Если $G$ не является $m$-группой, mо $|G|=p^{5} u|Z|=p$.

ДоКАЗАТЕЛЬСтво. Пусть $C(x)$ - неабелев собственный централизатор групшы $G$. Тогда $v(C(x)) \leqslant 4$. Поэтому из $|C(x)| \leqslant p^{4}$ и $\left|C^{2}(x)\right| \geqslant p^{2}$ получаем $|Z|=p$ и по лемме $1.1|G|=p^{5}$.

Лемма 2.2. Пусть $G$ - конечная неабелева $p$-группа, $v(G)=4,|Z|=p$. Тогда либо $G$ - центральное склеивание двух неабелевых групп порядка $p^{3}$, либо ступень нильпотентности группь $G$ больше двух. 
ДокАЗАТЕЛЬСтво. Пусть $G$ - двуступенно нильпотентна. Тогда для любого $x \in$ $G \backslash Z$ имеем $|C(x)|=|G: Z|=p^{4}$. Если $[x, y] \neq 1$, то из $G=C(y) \cdot C^{2}(x)$ получаем $\left|C^{2}(x): C(y) \cap C^{2}(x)\right|=p$. Из максимальности $C(x)$ следует, что $C^{2}(x)$ - минимальный централизатор в груше $G$. Поэтому $C^{2}(x) \cap C(y)=Z$. Итак, для любого элемента $x \in G \backslash Z$ вьполняются равенства $|G: C(x)|=\left|C^{2}(x): Z\right|=p$. По [7, лемма 11 и теорема 3] $G$ - центральное склеивание двух неабелевых групп порядка $p^{3}$.

Теорема 2.3. Конечная $p$-группа $G$, не являющаяся ни $m$-группой, ни чентральнымм склеиванием двух неабелевых групп порядка $p^{3}$, в том и только том случае удовлетворяет условию $v(G)=4$, когда $|G|=p^{5}$ u $G$ изоклинна одной из следующux грynn:

$$
\begin{aligned}
& G_{1}=\left\langle b_{1}\right\rangle\left\langle\left\langle b_{2}\right\rangle, b_{1}^{p^{3}}=b_{2}^{p^{2}}=1,\left[b_{1}, b_{2}\right]=b_{1}^{p}\right. \\
& G_{2}=\left(\left(\langle z\rangle \times\left\langle a_{1}\right\rangle \times\left\langle a_{2}\right\rangle\right) \lambda\left\langle b_{1}\right\rangle\right) \lambda\left\langle b_{2}\right\rangle, z^{p}=a_{i}^{p}=b_{i}^{p}=1, z \in Z,\left[a_{i}, b_{i}\right]=1, \\
& i=1,2 ;\left[a_{i}, b_{j}\right]=z n p u i \neq j,\left[b_{1}, b_{2}\right]=a_{1}, p \neq 2 ; \\
& G_{3}=((\langle z\rangle \times\langle x\rangle \times\langle a\rangle) \lambda\langle b\rangle) \lambda\langle c\rangle, z^{p}=x^{p}=a^{p}=b^{p}=c^{p}=1, z \in Z,[x, b]=1, \\
& {[x, c]=z=[a, b],[a, c]=x,[b, c]=a, p>3 .}
\end{aligned}
$$

ДокАЗАТЕльство. Пусть $G$ - конечная неабелева $p$-группа, $v(G)=4$ и $G$ не является ни $m$-группой, ни центральным склеиванием двух групп порядка $p^{3}$. Как показано выше, $|G|=p^{5},|Z|=p$, ступень нильпотентности $G$ больше двух и если $x \in Z_{2} \backslash Z$, то $|C(x)|=p^{4}$. Так как $G$ не $m$-групша, то $C(x)$ неабелев.

Если $y \in Z_{2} \backslash Z$ и $C(x) \neq C(y)$, то из соотношений порядков получим $|C(x) \cap C(y)|=p^{3}$. Если при этом $H=C(x) \cap C(y)$ неабелева, то $G=H \cdot C(H)$ нильпотентна ступени 2. Следовательно, $H$ абелева. Но тогда $H$ - максимальная абелева подгруппа как в $C(x)$, так и в $C(y)$, т.е. $y \in C(x)$. Это означает, в частности, что $Z_{2}-$ абелева подгрупша.

Рассмотрим сначала случай, когда $\left|Z_{2}\right|=p^{3}$. Тогда $Z_{2}=C\left(Z_{2}\right)$ и $G / Z_{2}=\left\langle\bar{b}_{1}\right\rangle \times\left\langle\bar{b}_{2}\right\rangle-$ элементарная абелева группа порядка $p^{2}$. В частности, $Z_{2} / Z$ не циклическая. Пусть $\left\langle\bar{b}_{i}\right\rangle=Z_{2}\left\langle b_{i}\right\rangle$ и $\left[b_{1}, b_{2}\right]=a_{1}$. В качестве образующего $z$ для $Z$ возьмем $\left[a_{1}, b_{2}\right]$ (если $\left[a_{1}, b_{2}\right]=1$, то поменяем нумерацию у $b_{1}$ и $\left.b_{2}\right)$. А элемент $a_{2}$ выберем так, чтобы выполнялись условия $a_{2} \in C\left(b_{2}\right) \cap Z_{2}$ и $\left[a_{2}, b_{1}\right]=z$. Если $\left[a_{1}, b_{1}\right]=z^{\alpha} \neq 1$, то заменим $b_{1}$ на $b_{2}^{-\alpha} b_{1}$. В результате мы получим, что в группе $G$ выполняются следующие равенства: $\left[b_{1}, b_{2}\right]=a_{1} ;\left[b_{i}, a_{i}\right]=1, i=1,2 ;\left[a_{i}, b_{j}\right]=z$ при $i \neq j,\left[a_{i}, a_{j}\right]=1$. Из этих равенств следует, что $\left[b_{1}^{p}, b_{2}\right]=a_{1}^{p}$ и $\left[b_{1}, b_{2}^{p}\right]=a_{1}^{p} z^{p(p-1) / 2}$. Поэтому если $p=2$, то $b_{2}^{2} \notin Z$. Кроме того, если $b_{1}^{p} \notin Z$, то $a_{1}^{p} \neq 1$, если $b_{2}^{p} \notin Z$, то $a_{1}^{p} \neq 1$, и, наконец, если $a_{1}^{p} \neq 1$, то $b_{1}^{p} \notin Z$ и $b_{2}^{p} \notin Z$. Как нетрудно видеть, группа $G$ изоклинна одной из групп $G_{1}, G_{2}$.

Предположим теперь, что $\left|Z_{2}\right|=p^{2}, x \in Z_{2} \backslash Z, Z=\langle z\rangle$, а $y$ - элемент из $G$ с неабелевым централизатором. Тогда $|C(y)|=p^{4}$ и, как и вьше, $y \in C(x)$. Следовательно, для любого $y \notin C(x)$ подгруппа $C(y)$ абелева. Так как $|C(y)| \leqslant p^{3}$, то $C(y) \cap C(x)-$ абелева подгрупша порядка не больше $p^{2}$. Если $a \in(C(y) \cap C(x)) \backslash Z$, то $C(a)$ содержит $x$ и $y$, т.е. неабелев. Но тогда $a \in Z_{2} \cap C(y)=Z$, что невозможно. Итак, в этом случае $C(x)$ - единственный неабелев централизатор и для всех $y \notin C(x)$ подгруппа $C(y)$ абелева порядка $p^{2}$.

Пусть ступень нильпотентности группы $G$ равна $3, c \in G \backslash C(x),[x, c]=z, a$ и $b$ - неперестановочные элементы из $C(x)$. Пусть также $[a, c]=x^{\alpha_{1}} z^{\alpha_{2}},[b, c]=x^{\beta_{1}} z^{\beta_{2}}$. Если $\alpha_{1} \equiv 0(p)$, то $\alpha_{2}$ не делится на $p$. Пусть $s \alpha_{2} \equiv 1(p)$. Тогда $\left[a^{s}, c\right]=[a, c]^{s}=z=[x, c]$, т.е. $a^{s} x^{-1} \in C(c)$, что невозможно. Поэтому $\alpha_{1}$ не делится на $p$. Аналогично, $\beta_{1}$ не делится на $p$. Но тогда если $\alpha_{1}+s \beta_{2} \equiv 0(p)$, то $\left[a b^{s}, c\right] \in Z$, и снова получим противоречие. 
Итак, ступень нильпотентности групшы $G$ равна 4. Тогда, в тех же обозначениях, можно считать, что $Z_{3}(G)=\langle z, x, a\rangle=C(a)$. В самом деле, так как $Z_{3} / Z_{2}$ - циклическая группа простого порядка, то из $C(x) / Z_{2} \triangleleft G / Z_{2}$ следует, что $Z_{3} \leqslant C(x)$. Пусть $[b, c]=a,[x, c]=z,[a, b]=x^{\alpha_{1}} z^{\alpha_{2}},[a, c]=x^{\beta_{1}} z^{\beta_{2}}$. Если $\beta_{1} \equiv 0(p)$ и $s \beta_{2} \equiv 1(p)$, то снова получим $a^{s} x^{-1} \in C(c)$, что невозможно. Поэтому $\beta_{1}$ не делится на $p$. Если $\alpha_{1}$ не делится на $p$ и $s \alpha_{1}+\beta_{1} \equiv 0(p)$, то $b^{s} c \in C(a)$, что тоже невозможно, т.е. $\alpha_{1} \equiv 0(p)$, и мы можем считать, что $[a, b]=z$.

Если положить $z^{\prime}=z^{\beta_{1}^{2}}, x^{\prime}=x^{\beta_{1}^{2}} z^{\beta_{1} \beta_{2}}, a^{\prime}=a^{\beta_{1}} z^{\beta_{1}\left(\beta_{1}-1\right) / 2}$ и $b^{\prime}=b^{\beta_{1}}$, то получим $\left[b^{\prime}, c\right]=a^{\prime},\left[x^{\prime}, c\right]=z^{\prime},\left[a^{\prime}, b^{\prime}\right]=z^{\prime}$ и $\left[a^{\prime}, c\right]=x^{\prime}$. Поэтому будем в дальнейшем считать, что $[a, c]=x$.

Подгруппа $G^{\prime}=\langle z, x, a\rangle$ - абелева группа порядка $p^{3},|Z|=p$. Предположим, что $x^{p} \neq 1$. Тогда $C^{2}(x)$ - циклическая группа порядка $p^{2}$ и $x^{p} \in Z$. Из $\left\langle\left[a^{p}, c\right]\right\rangle=\left\langle x^{p}\right\rangle$ получаем, что $a^{p} \notin Z$. Но тогда из $|\langle a, x\rangle|=p^{3}$ следует, что $\langle a\rangle \cap\langle x\rangle \neq 1$, т.е. $a^{p} \in Z$. Противоречие.

Таким образом, $x^{p}=1$ и $Z_{2}=\langle z\rangle \times\langle x\rangle$. Так как $|C(c)|=p^{2}$, то $c^{p} \in Z$. Из $\left[b, c^{p}\right]=a^{p} x^{p(p-1) / 2} z^{p(p-1)(p-2) / 6}=1$ следует, что $a^{p}=1$ и $p>3$. Несложно проверить, что $b^{p} \in Z$. Поэтому $G$ изоклинна группе $G_{3}$.

В следующей теореме $p, q, r$ - различные простые числа.

ТЕОрема 2.4. Пусть $G$ - конечная ненильпотентная группа, $Z_{2}(G) \neq Z(G) u$ $G$ не является m-группой. Тогда $v(G)=4$ в том и только том случае, когда выполняется одно из следующих условий:

1) $G=P \times Q$, әде $P$ и $Q$-неабелевы группы порядков $p^{3}$ и qr соответственно;

2) $G=(\langle a\rangle \lambda\langle b\rangle) \lambda P, P$ - неабелева группа порядка $p^{3}, a^{q}=b^{r}=1,[a, b] \neq 1$, $q-1$ делится на $p r,[b, P]=1,|C(a)|=q p^{2}$

3) $G=P \times Q$, где $P$ и $Q$ - неабелевы группы порядков $p^{3}$ и $p q$ соответственно;

4) $G=\langle b\rangle \lambda(\langle y\rangle \lambda\langle x\rangle), b^{q}=x^{p}=y^{p^{3}}=1, b^{x}=b, b^{y}=b^{r}, r^{p^{2}} \equiv 1(q), r^{p} \not \equiv 1(q)$, $[x, y]=y^{p^{2}}$

5) $G=\langle b\rangle \lambda(\langle x\rangle \lambda\langle y\rangle), b^{q}=x^{p^{2}}=y^{p^{2}}=1, b^{x}=b, b^{y}=b^{r}, r^{p^{2}} \equiv 1(q), r^{p} \not \equiv 1(q)$, $[x, y]=x^{p}$

6) $G=\langle b\rangle \lambda[(\langle z\rangle \times\langle x\rangle) \lambda\langle y\rangle], b^{q}=x^{p}=z^{p}=y^{p^{2}}=1, z \in Z, b^{x}=b, b^{y}=b^{r}$, $r^{p^{2}} \equiv 1(q), r^{p} \not \equiv 1(q),[x, y]=z$

7) $|G|=p^{4} q$ и $G$ изоклинна одной из следующих групп:

a) $(\langle b\rangle \times[(\langle z\rangle \times\langle x\rangle) \lambda\langle y\rangle]) \lambda\langle a\rangle, b^{q}=z^{p}=x^{p}=a^{p}=y^{p}=1, z \in Z, b^{a}=b^{r}$, $r^{p} \equiv 1(q), r \not \equiv 1(q),[y, a]=[x, a]=1,[x, y]=z$;

б) $\langle b\rangle \lambda(\langle a\rangle \lambda\langle y\rangle), b^{q}=a^{p^{3}}=y^{p}=1, b^{y}=b, b^{a}=b^{r}, r^{p} \equiv 1(q), r \not \equiv 1(q)$, $[a, y]=a^{p}$

в) $\langle b\rangle \lambda[(\langle z\rangle \times\langle x\rangle \times\langle a\rangle) \lambda\langle y\rangle], b^{q}=z^{p}=x^{p}=a^{p}=y^{p}=1, z \in Z, b^{x}=b^{y}=b$, $b^{a}=b^{r}, r^{p} \equiv 1(q), r^{p} \not \equiv 1(q),[a, y]=x,[x, y]=z$.

ДокАЗАТЕЛьство. Пусть $G$ - ненильпотентная групша, $v(G)=4$ и $Z_{2} \neq Z$. Если $x \in Z_{2} \backslash Z$, то снова получим $|G|=|C(x)| \cdot|I|$, где $I$ - образ гомоморфизма $f: g \rightarrow[g, x]$.

Если $w(Z) \geqslant 2$, то $w\left(C^{2}(y)\right) \geqslant 3$ для любого $y \in G \backslash Z$, т.е. все собственные централизаторы в групше $G$ абелевы. Поэтому $|Z(G)|=p$ для некоторого простого числа $p$. Так как $Z=I \geqslant G^{\prime}$, то по теореме VI.14.3 из [9] силовская $p$-подгруппа $P$ группы $G$ неабелева, т.е. $|P| \geqslant p^{3}$. Из $w(C(x)) \leqslant 4$ следует $w(G)=5$ и $w(P) \leqslant 4$. Так как $\left[x^{p}, G\right]=1$, 
то $x$ является $p$-элементом, т.е. $x \in P$. Предположим сначала, что $|P|=p^{3}$. Обозначим через $Q$ холлову $p^{\prime}$-подгруппу из $G$. Тогда $w(Q)=2$ и $|C(x)|=p^{2}|Q|$. Поэтому $C(x)=C_{P}(x) \times Q, Q \triangleleft G$ и $G=Q \lambda P$.

Так как $G$-не $m$-группа и $|G / C(x)|=p$, то $C(x)$ неабелев, т.е. $Q$-неабелева подгруппа порядка $q r$, где $q, r$ - различные простые числа, отличные от $p$. Пусть $Q=\langle a\rangle \lambda\langle b\rangle$, $|a|=q,|b|=r$, и $y \in P \backslash C(x)$.

Так как $\langle a\rangle \lambda\langle b\rangle$ не нильпотентна, то по теореме Томпсона (см. теорему V.8.7.a) из [9]) о нильпотентности групшы, допускаюшей регулярную групш автоморфизмов, $y$ действует на $\langle a\rangle \lambda\langle b\rangle$ нерегулярно, т.е. существует неединичный элемент $c \in\langle a\rangle \lambda\langle b\rangle$ такой, что $c^{y}=c$. Предположим сначала, что $c$ сопряжен со степенью $b$. Можно считать, что $y \in C(b)$. Если $y \in C(a)$, то $G=Q \times P$. Если же $y \notin C(a)$, то $(q-1)$ делится на $p r$, $C(a)=\langle a\rangle \times C^{2}(x)$ и $G / C(a)$ - циклическая группа порядка $p r$.

Пусть теперь $c=a$. Так как подгрупп, сопряженных с $\langle b\rangle$, ровно $q$, то $P \leqslant N(\langle b\rangle)$. Из $y \in N(\langle b\rangle)$ следует, что $y^{a}=y \in N\left(\left\langle b^{a}\right\rangle\right)$. Пусть $b^{a}=b a^{s}$ и $b^{y}=b^{t}$. Тогда $\left(b a^{s}\right)^{y}=b^{y} a^{s}=b^{t} a^{s} \in\left\langle b a^{s}\right\rangle$, т.е. $b^{t-1} \in\left\langle b^{a}\right\rangle$. Отсюда $t=1$, т.е. $y \in C(b)$ и $G=Q \times P$.

Итак, в случае $|P|=p^{3}$ мы получаем группы 1$\left.), 2\right)$. Пусть теперь $|P|=p^{4}$. Так как $|C(x)|=p^{3} q,\left|C^{2}(x)\right| \geqslant p^{2}$ и $G$ не является $m$-группой, то $C(x) / C^{2}(x)$ - неабелева группа порядка $p q$. Пусть $Q$ - силовская $q$-подгруппа из $G$. Из $C(x) \triangleleft G$ следует, что либо $C^{2}(x) \times Q \triangleleft G$ и, следовательно, $Q \triangleleft G$, либо $P \cap C(x) \triangleleft G$.

Предположим сначала, что $P \cap C(x) \triangleleft G$. Из $|P \cap C(x)|=p^{3}$ и $\left|C^{2}(x)\right| \geqslant p^{2}$ следует, что $P \cap C(x)$ абелева. По [10, следствие 0.5] $C(x)=C^{2}(x) \times(\langle a\rangle \lambda\langle b\rangle),|a|=p$, $|b|=q$. Так как $C(x) \triangleleft G$ и $(C(x))^{\prime}=\langle a\rangle$, то $\langle a\rangle \triangleleft G$ и, следовательно, $a \in Z(P)$. Из $H=\left(C^{2}(x) \times\langle a\rangle\right) \triangleleft G$ и $|G / H|=p q$ следует, что $P \triangleleft G$.

Все силовские $q$-подгрупшы группы $G$ содержатся в $C(x)$, и число их равно $p$. Поэтому $N(\langle b\rangle)$ содержит элемент $y \notin C(x)$. Ясно, что $y \in C(b)$. Но тогда $y \in C(\langle a\rangle \lambda\langle b\rangle)$ и

$$
G=(\langle a\rangle \lambda\langle b\rangle) \cdot C(\langle a\rangle \lambda\langle b\rangle) .
$$

Так как $a \notin C(\langle a\rangle \lambda\langle b\rangle)$, то $P_{1}=\left\langle C^{2}(x), y\right\rangle=C(\langle a\rangle \lambda\langle b\rangle)$ - неабелева групша порядка $p^{3}$ и $G=P_{1} \times(\langle a\rangle \lambda\langle b\rangle)$, т.е. $G$ группа типа 3$)$.

Пусть теперь $Q \triangleleft G, G=Q \lambda P$. Тогда $C(x)=Q \lambda C_{P}(x)$ и $C_{P}(x)$ - абелева подгруппа порядка $p^{3}$. Так как $C(x)$ неабелев, то $C(x)=\left(Q \times C^{2}(x)\right)\langle a\rangle, Q \lambda\langle a\rangle$ неабелева. Из $C(Q) \geqslant Q \times C^{2}(x)$ следует, что либо $|C(Q)|=q p^{2}$, либо $|C(Q)|=q p^{3}$.

Предположим сначала, что $|C(Q)|=q p^{2}$, т.е. $C(x)=C(Q)\langle a\rangle$. Так как $G / C(Q)-$ циклическая группа, то $a=y^{p}$ для некоторого $y \notin C(x)$. Но тогда $a \in Z(P)$, т.е. $|Z(P)|=p^{2}$. В этом случае, как несложно видеть, группа $G$ изоморфна одной из групा 4)-6).

Пусть теперь $|C(Q)|=q p^{3}$. Тогда $C(Q)=Q \times P_{1}$, где $P_{1}$ - неабелева группа порядка $p^{3}, P=P_{1}\langle a\rangle$. Если $|Z(P)|>p$, то $P=C(Z(P))$ и $P_{1}=C(Q) \cap P$ является централизатором. Но тогда $G=P_{1} \cdot C\left(P_{1}\right)=P_{1} \cdot(Q \lambda\langle a\rangle)$. В этом случае $G^{\prime}=Q \times Z$ и $G / Z \cong\langle b\rangle \lambda(\langle x\rangle \times\langle y\rangle \times\langle a\rangle), b^{q}=x^{p}=y^{p}=a^{p}=1, b^{x}=b^{y}=b, b^{a}=b^{r}, r^{p} \equiv 1(q)$, $r \not \equiv 1(q)$, т.е. $G$ изоклинна группе типа $7 \mathrm{a})$.

Пусть теперь $|Z(P)|=p$, т.е. $Z(P)=Z(G)$. В этом случае $P$ нильпотентна ступени 3. В самом деле, предположим, что $P$ двуступенно нильпотентна, $y \in P \backslash C(x)$. Тогда можно считать, что $[x, y]=[a, y]=z$. Отсюда $x a^{-1} \in C(y)$ и, следовательно, $x a^{-1} \in Z(P)$, что невозможно.

Несложно проверить, что в этом случае $G$ изоклинна групе 7б) или 7в). 


\section{3. Группы с условием $Z_{2}=Z>1$.}

Лемма 3.1. Если $Z_{2}(G)=Z(G)$ и $Z$ не лежит в $G^{\prime}$, то $v(G / Z) \leqslant v(G)-1$.

ДокАЗАТЕЛЬСТво. Пусть $h$ является элементом из $G \backslash Z$ с наибольшим значением $w\left(C_{G / Z}(h Z)\right)$. Обозначим $L / Z=C_{G / Z}(h Z)$. Если $f$ - гомоморфизм из $L$ в $Z \cap L^{\prime}$ такой, что $f(l)=[l, h]$, и $I-$ его образ, то $L / C(h) \cong I$. Поэтому

$$
|L / Z|=|L / C(h)| \cdot|C(h) / Z|=|C(h)| \cdot|I| /|Z| .
$$

Так как $Z$ не лежит в $G^{\prime}$, то $|I|<|Z|$ и

$$
v(G / Z)=w(L / Z) \leqslant w(C(h))-1 \leqslant v(G)-1 .
$$

Теорема 3.2. Пусть $G$ - конечная неразрешимая группа, $Z_{2}(G)=Z(G) \neq 1$ и $G$ не является $m$-группой. Тогда в группе $G$ выполняется условие $v(G)=4$ в том и только том случае, когда $G$ изоморфна одной из следующих групп:

1) $G=\langle a\rangle \times H,|a|-$ простое число, $H \cong S_{5}$ или $H \cong P S L(2, q), q \in\left\{5^{3}, 3^{3}, 3^{2}, p\right\}$, где $p$ - простое число со свойством $l(p)=3$;

2) $G=H \lambda\langle a\rangle, H \cong A_{5}, a^{4}=1, a^{2} \in Z u G /\left\langle a_{2}\right\rangle \cong S_{5}$;

3) $G / Z \cong P S L(2,9)$ или $P G L(2,9),|Z|=3$ u $G^{\prime} \cong P S L(2,9)^{*} /\left\langle k^{2}\right\rangle$, zде $\langle k\rangle-$ мультипликатор Шура группь $P S L(2,9)$;

4) $G \cong A_{7}^{*} /\left\langle k^{2}\right\rangle$, где $\langle k\rangle-$ мультипликатор Шура группы $A_{7}$.

ДокАЗАТЕЛЬСТво. Пусть $G$ - группа из условия теоремы. Так как $G$ не является $m$-группой, то $|Z(G)|=p$ - простое число.

Предположим сначала, что $Z \not G^{\prime}$. Тогда по лемме $3.1 v(G / Z)=3$. Так как $G / Z$ имеет тривиальньй центр, то по теореме 4.1 из [2] и замечанию, сделанному после теоремы 1.1 из [3], либо $G / Z \cong S_{5}$, либо $G / Z \cong P S L(2, q)$, где $q$ определено в п. 1) теоремы.

Если $G / Z \cong P S L(2, q)$, то из $(G / Z)^{\prime}=G / Z$ получаем $G=G^{\prime} \cdot Z=G^{\prime} \times Z$. Предположим теперь, что $G / Z \cong S_{5}$. Если $Z \not \Phi(G)$ и $H$ - максимальная подгруппа группы $G$, не содержащая $Z$, то $G=Z \times H$ и $H \cong S_{5}$. Пусть $Z \leqslant \Phi(G)$ и $H=G^{\prime} Z$. Так как $Z \not G^{\prime}$, то $H=G^{\prime} \times Z$ и $G^{\prime} \cong A_{5}$. Кроме того, $|G: H|=2$. Пусть $a-$ элемент из $G \backslash H$. Тогда $G=G^{\prime}\langle a\rangle$. Подгруппа $H$ не содержит элементов порядка 4 . Поэтому $|a|=4$, $G=G^{\prime} \lambda\langle a\rangle$ и $|Z|=2$. Так как $a Z / Z$ имеет порядок 2 , то $a^{2} \in Z$.

Предположим теперь, что $Z \leqslant G^{\prime}$. В силу [2] и теорем 1.1 и 1.2 из [3] $G / Z$ изоморфна либо группе $P S L(2, q), q \in\left\{2^{4}, 7^{3}, 5^{3}, 3^{3}, 2^{3}, 5^{2}, 3^{2}, p\right\}$, где $l(p) \in\{3,4\}$, либо группе $P G L(2, q), q \in\left\{5^{3}, 3^{3}, 5^{2}, 3^{2}, p\right\}$, где $l(p) \in\{2,3\}$, либо групше из пункта 3) или 4) теоремы 1.2 из [3], либо $A_{7}$.

Если $G / Z \cong P S L(2, q)$, то из $G=G^{\prime}$ следует, что $G$ - накрьвающая для $P S L(2, q)$. Но для $P S L(2,16)$ и $P S L(2,8)$ мультипликатор Шура тривиален, а для $P S L(2, q), q \neq 9$ нечетно, накрывающей является групша $S L(2, q)$, которая по теореме 5.9 из [4] является $m$-группой. В случае $q=9$ мультипликатор Шура $\langle k\rangle$ имеет порядок 6 . Поэтому $|Z|=2$ или 3 . Если $|Z|=2$, то снова $G \cong S L(2,9)$, т.е. является $m$-группой. Следовательно, $|Z|=3$ и $Z=\left\langle k^{2}\right\rangle$.

Пусть $G / Z \cong P G L(2, q)$. В этом случае $G^{\prime} / Z \cong P S L(2, q)$ и $G^{\prime}$ - накрывающая для $P S L(2, q)$. Снова используя теорему 5.9 из [4], получим $q=9$ и $|Z|=3$. 
В случае группы $A_{7}$ мультипликатор Шура имеет порядок 6 . Так как порядок силовской 2-подгрупшы группы $A_{7}$ равен 16 , то $(|Z|, 2)=1$.

Рассмотрим теперь случай, когда $G$ изоморфна группе из пункта 3$)$ или 4$)$ теоремы 1.2 из [3]. Заметим, что если $[x, y] \in Z \backslash 1$, то из $\left[x^{p}, y\right]=\left[x, y^{p}\right]=[x, y]^{p}=1$ следует, что $x$ и $y$ являются $p$-элементами. Это означает, что если $x p^{\prime}$-элемент из $G$, то $C_{G / Z}(x Z)=C(x) / Z$, т.е. $w(C(x))=w\left(C_{G / Z}(x Z)\right)+1$. Поэтому $p=\exp (H / Z)$.

Пусть $\bar{H}=H / Z$ - инвариантная абелева подгруппа порядка $p^{3}$ из $G / Z$. Как отмечалось при доказательстве теоремы 1.2 из [3], $\bar{H}=\left\langle\overline{a_{1}}\right\rangle \times\left\langle\overline{a_{2}}\right\rangle \times\left\langle\overline{a_{3}}\right\rangle$, где $C_{\bar{G}}\left(\left\langle\overline{a_{i}}\right\rangle\right)=\bar{H} \lambda\left\langle\overline{\tau_{i}}\right\rangle$, $\overline{\tau_{i}} \in S_{2}(G / Z)$. Так как $w\left(C\left(a_{i}\right)\right) \leqslant 4$ и подгруппа $\left\langle a_{1}\right\rangle Z$ нормализуется $\tau_{2}$, то можно считать, что $\left[a_{1}, a_{2}\right]=z \neq 1$ и $a_{1}^{\tau_{2}}=a_{1}^{-1}$. Но тогда

$$
z=\left[a_{1}, a_{2}\right]^{\tau_{2}}=\left[a_{1}^{\tau_{2}}, a_{2}^{\tau_{2}}\right]=\left[a_{1}^{-1}, a_{2}\right]=z^{-1}
$$

что невозможно.

Для описания разрешимых групп с нетривиальным центром нам понадобится следующий результат.

Теорема 3.3 [2, теорема 3.4]. Если $G$ - группа без иентра, $v(G)=3 u G$ не является m-группой, то выполняется один из следующих случаев:

1) $G=P \lambda(\langle b\rangle \lambda\langle c\rangle)$ - әруппа Фробениуса, $P$ - әлементарная абелева поряд$\kappa a p^{3},|b|=q,|c|=r^{2}, q u r-$ nростые числа, $b^{c}=b^{\alpha}, \wp(\alpha)=r ;$

2) $G=H \times K, H$ и $K$ - неабелевы группь порядков $p_{1} q_{1}$ и $p_{2} q_{2}$ соответственHo;

3) $G=(\langle a\rangle \times\langle b\rangle) \lambda\langle c\rangle, a^{p}=b^{q}=c^{r s}=1, a^{c}=a^{\alpha}, \wp(\alpha)=r s, b^{c}=b^{\beta}, \wp(\beta)=r$;

4) $G=P \lambda(H \lambda\langle\tau\rangle), P$ - әлементарная абелева порядка $p^{2}, \tau^{2}=1,\left|C_{P}(\tau)\right|=p$, $|H|$ нечетен, $H \lambda\langle\tau\rangle$ действует на $P$ неприводимо и либо $H=\langle c\rangle, c^{\tau}=c^{-1}$, $w(H) \leqslant 3$, либо $H=\langle c\rangle \times\langle t\rangle,|c| u|t|-$ nростые числа, $c^{\tau}=c^{-1}, t^{\tau}=t ;$

5) $G=S_{4}$;

6) $G=P \lambda\langle c\rangle$ - әруппа Фробениуса, $P$ - неабелева группа порядка $p^{3}, w(|c|) \leqslant 3$;

7) $G=P \lambda Q$ - әруппа Фробениуса, $P$ - элементарная абелева порядка $p^{2}$ или $p^{3}, Q$ - группа кватернионов порядка 8;

8) $G=P \lambda Q, P$ - элементарная абелева порядка $p^{2}, p>2, Q-$ группа диэдра порядка 8 и иентральная инволюиия из $Q$ инвертирует все әлементы из $P$;

9) $G=P \lambda\langle c\rangle, w(|c|) \leqslant 2, P$ - неабелева порядка $p^{3}, C\left(c_{0}\right)=\langle a\rangle \lambda\langle c\rangle$, где $c_{0}$ некоторый элемент простого порядка из $\langle c\rangle, a$ - нецентральный элемент порядка р из $P$ и если $w(|c|)=2$, то $C(c)=\langle c\rangle$.

Отметим, что группы типа 9) в [2] определены неточно.

Условимся говорить, что в групе $H$ элемент $y$ вырастает, если в определяющих соотношениях группы $H$ соотношение $y^{n}=1$ заменяется соотношением $y^{n p}=1$, где $p$ - простой делитель числа $n$. Кроме того, будем говорить, что элемент $z$ врастает в әрynny $H$, если в группе $H$ элементарная абелева подгруппа $\langle a\rangle \times\langle b\rangle$ заменяется на неабелеву групшу порядка $p^{3}$ вида $(\langle z\rangle \times\langle a\rangle) \lambda\langle b\rangle$.

Нам понадобится следующий результат о группах порядка $p^{4}$ (см. $[9$, III, 12.6]): если $|F|=p^{4}$ и $|Z(F)|=p$, то $F$ - одна из следующих групп:

а) $F=(\langle a\rangle \times\langle b\rangle) \lambda\langle x\rangle, a^{p^{2}}=b^{p}=x^{p}=1,[a, x]=b,[b, x]=a^{s p}$, где $s=1$ или $s-$ квадратичньй невычет по модулю $p$; 
б) $F=(\langle z\rangle \times\langle a\rangle \times\langle b\rangle) \lambda\langle x\rangle, z^{p}=a^{p}=b^{p}=x^{p}=1,[z, x]=1,[a, x]=z,[b, x]=a$;

в) $F=(\langle a\rangle \lambda\langle b\rangle) \lambda\langle x\rangle, a^{p^{2}}=b^{p}=x^{p}=1,[a, b]=a^{p},[a, x]=b,[b, x]=1$ при $p>3$ и $F=(\langle a\rangle \times\langle b\rangle)\langle x\rangle, a^{9}=b^{3}=1, a^{3}=x^{3},[a, x]=b,[b, x]=a^{3}$ при $p=3$.

Теорема 3.4. Пусть $G$ - конечная разрешимая группа, не являющаяся $m$-группой, $и Z_{2}=Z>1$. Тогда условие $v(G)=4$ выполняется тогда и только тогда, когда $G$ - одна из следующих групп:

1) $G=Z \times G_{1},|Z|$ - простое число, $G_{1}$ - одна из групп 1) - 9) теоремы 3.3 ;

2) $G$ получается из групn 1),3),6),9) теоремы 3.3 за счет вырастания әлементас;

3) $G$ получается за счет вырастания неинвариантного мнохсителя из $Н$ или $K$ в случае әруппы 2), әлемента $\tau$ или әлемента $t$ в случае әруппы 4), инволючии, не лежащей в $A_{5}$, в случае группь 5), нечентральной инволюиии из $Q$ в группе 8), неединичного әлемента из $P \cap C(c)$ в группе 9), если $w(|c|)=1$

4) $G=A \lambda B, A$ - әлементарная абелева группа порядка $p^{2}, B$ - неабелева группа порядка $q^{3}, Z=Z(B)$ u $G / Z$ - группа типа 2) из теоремы 3.3 ;

5) $G=P \lambda Q, P$ - неабелева группа порядка $p^{3}$ и әкспоненты $p, Q$ - группа кватернионов порядка $8, Z=Z(P)$ u $G / Z$ - группа Фробениуса;

6) $G=P \lambda Q, P$ - абелева группа порядка $p^{2}, Q$ - группа кватернионов, группа диэдра или квазидиэдральная группа, $|Q|=16, Z=Z(Q)$ u $G / Z$ - әруппа muпа 8) из теоремы 3.3;

7) $G=((\langle z\rangle \times\langle a\rangle \times\langle b\rangle) \lambda\langle x\rangle) \lambda\langle c\rangle, z^{p}=a^{p}=b^{p}=x^{p}=1,[z, x]=1,[a, x]=z$, $[b, x]=a,|c|$ нечетен, $w(|c|)=3,[z, c]=1, a^{c}=a^{\alpha}, \wp(\alpha)=|c|, b^{c}=$ $a^{\alpha(\alpha-1) / 2} b^{\alpha^{2}}, x^{c}=a^{\delta} x^{\alpha^{-1}}$

8) $G=F \lambda K, F-$ иентральное склеивание двух неабелевы $K=Q_{8}$ или $K=\langle a\rangle \lambda\langle b\rangle, a^{q_{1}}=b^{q_{2}^{2}}=1,\left[a, b^{q_{2}}\right]=1,[a, b] \neq 1, Z=Z(F) u$ $G / Z$ - әруппа Фробениуса;

9) $G=\left(\left\langle b_{1}\right\rangle \times\left\langle b_{2}\right\rangle\right) \lambda\left(\left(\left(\left\langle a_{1}\right\rangle \times\langle z\rangle\right) \lambda\left\langle a_{2}\right\rangle\right) \times\left\langle a_{3}\right\rangle\right), b_{1}^{p}=b_{2}^{p}=a_{1}^{q}=a_{2}^{q}=a_{3}^{r}=1$, $q \neq r,\left[a_{1}, a_{2}\right]=z,\left[a_{1}, b_{1}\right]=\left[a_{2}, b_{2}\right]=1,\left\langle b_{1}\right\rangle \lambda\left\langle a_{2} a_{3}\right\rangle u\left\langle b_{2}\right\rangle \lambda\left\langle a_{1} a_{3}\right\rangle-$ zpynnbl Фробениуса;

10) $G=\left(\left\langle b_{1}\right\rangle \times\left\langle b_{2}\right\rangle\right) \lambda H, w(H)=5, H=\langle a\rangle \lambda Q,|a|$ - нечетный делитель числа $p+1$ и либо $Q=\langle x\rangle\langle y\rangle$ - группа кватернионов порядка 8, 16 или 32, $y^{4}=1$, либо $Q=\langle x\rangle \lambda\langle y\rangle-$ диәдральная или квазидиэдральная группа порядка 8, 16 или 32. В любом случае $b_{1}^{y}=b_{2}, b_{2}^{y}=b_{1}, a^{y}=a^{p},[a, x]=1 u\langle a x\rangle$ действует на $\left\langle b_{1}\right\rangle \times\left\langle b_{2}\right\rangle$ неприводимо;

11) $G=F \lambda\langle d\rangle, F-$ zруппа типа $G_{2}$ или $G_{3}$ из теоремы $2.3, w(|d|) \leqslant 3, Z=Z(F)$ u $G / Z$ - әруппа Фробениуса.

ДокАЗАтЕЛьСтво. Пусть $G$ - группа из условия теоремы. Тогда $|Z|$ - простое число. Если $G / Z$ является $m$-группой, то $G / Z$ - группа Фробениуса с абелевьпии инвариантным и дополнительным множителями. Но тогда по теореме 5.12 из [3] и $G$ должна быть $m$-группой. Поэтому $G / Z$ не является $m$-группой.

Если $Z \nless \Phi(G)$, то $G=Z \times G_{1}-$ группа типа 1 .

Предположим, что $Z \leqslant \Phi(G)$, но $Z \nless G^{\prime}$. В силу леммы $3.1 G / Z$ является группой из теоремы 3.3. В этом случае несложные рассуждения показывают, что $G$ является группой типа 2 или 3. 
Пусть теперь $Z \leqslant \Phi(G) \cap G^{\prime}$. Рассмотрим сначала случай $v(G / Z)=3$. Тогда $G / Z-$ группа из теоремы 3.3. Исследуем каждый из девяти случаев этой теоремы.

1) Элемент $z$ может врасти только в подгруппу $P$. Но тогда $G=F \lambda K$ и $F=\left(\left(\left\langle a_{1}\right\rangle \times\right.\right.$ $\left.\langle z\rangle) \lambda\left\langle a_{2}\right\rangle\right) \times\left\langle a_{3}\right\rangle$. Так как подгруппы $\langle z\rangle$ и $\langle z\rangle \times\left\langle a_{3}\right\rangle K$-допустимы, то можно считать, что и $\left\langle a_{3}\right\rangle$ тоже $K$-допустима. Но тогда фактор-групша $G / F=G / C\left(a_{3}\right)$ должна быть циклической, что не вьполняется.

2) Пусть $G / Z=\left(\left\langle\bar{a}_{1}\right\rangle \times\left\langle\bar{a}_{2}\right\rangle\right) \lambda\left(\left\langle\bar{b}_{1}\right\rangle \times\left\langle\bar{b}_{2}\right\rangle\right),\left[\bar{a}_{i}, \bar{b}_{j}\right]=1$ при $i \neq j,\left\langle\bar{a}_{i}\right\rangle \lambda\left\langle\bar{b}_{i}\right\rangle-$ групшы $\Phi$ робениуса порядков $p_{i} q_{i}$. Если $z$ врастает в $\left\langle\bar{a}_{1}\right\rangle \times\left\langle\bar{a}_{2}\right\rangle$, то

$$
z=z^{b_{1}}=\left[a_{1}^{b_{1}}, a_{2}^{b_{1}}\right]=\left[a_{1}^{b_{1}}, a_{2}\right]=z^{\alpha}
$$

для $\alpha \not \equiv 1(p)$. Поэтому $z$ врастает в $\left\langle\bar{b}_{1}\right\rangle \times\left\langle\bar{b}_{2}\right\rangle$, и мы получаем группу типа 4 .

3) В этом случае $p=q$ и $z$ врастает в $\langle\bar{a}\rangle \times\langle\bar{b}\rangle$. Но тогда $z=z^{c}=\left[a^{c}, b^{c}\right]=z^{\alpha \beta}$, т.е. $\alpha \beta=1$, что противоречит тому, что $\wp(\alpha)=r s, \wp(\beta)=r$.

4) Если $z$ врастает в $H$, то $|\bar{c}|=|\bar{t}|=|z|$ и из равенства $z=[c, t]$ получим $z=z^{\tau}=$ $\left[c^{-1}, t\right]=z^{-1}$, что невозможно. Если же $z$ врастает в $P$, то $F=(\langle a\rangle \times\langle z\rangle) \lambda\langle b\rangle$, и так как $\tau$ действует на $P$ не регулярно, то можно считать, что $a^{\tau}=a, b^{\tau}=b^{\alpha}, \alpha \neq 1$. Но тогда снова $z=\left[a, b^{\alpha}\right]=z^{\alpha}$, что невозможно.

5) Накрьвающая для $S_{4}$ удовлетворяет условию $v(G)=3$.

6) Элемент $z$ врастает в $P$. Так как $|F|=p^{4}$ и $F /\left(Z(F) \cap F^{\prime}\right)$ неабелева, то $F$ может быть только одной из групп а)-в), перечисленных перед формулировкой теоремы. Случаи а) и в) невозможны, так как в этих случаях $F$ содержит $c$-допустимую циклическую подгруппу $\langle a\rangle$ порядка $p^{2}$ и, следовательно, должно выполняться равенство $\langle a\rangle=Z \times[a,\langle c\rangle]$.

Пусть $F$ - группа типа б). Тогда можно считать, что $z^{c}=z, a^{c}=a^{\alpha}, b^{c} \equiv a^{\beta} b^{\gamma}$ и $x^{c} \equiv a^{\delta} b^{\varepsilon}(\bmod Z)$. Действуя на равенства $[a, x]=z$ и $[b, x]=a$ элементом $c$, получим $\varepsilon=\alpha^{-1}, \gamma=\alpha^{2}$ и $\beta=\alpha(\alpha-1) / 2$, т.е. $G$ - группа типа 7 .

7) Так как мультипликатор Шура для группы кватернионов тривиален, то $z$ врастает в $P$ и мы имеем групу типа 5 .

8) Пусть $z$ врастает в $P$. Тогда $F=(\langle a\rangle \times\langle z\rangle) \lambda\langle b\rangle$. Так как нецентральная инволюция $\tau$ из $Q$ действует на $P$ не регулярно, то можно считать, что $a^{\tau}=a, b^{\tau}=b^{-1}$. Но тогда $z=z^{\tau}=z^{-1}$, что невозможно. Таким образом, $z$ врастает в $Q$. Так как мультипликатор Шура диэдральной 2-групшы имеет порядок 2 , а накрывающая ее является кватернионной, диэдральной или квазидиэдральной групой, то $G$ - группа типа 6 .

$9)$ В этом случае $z$ врастает в $P$ и $F$ снова одна из групп а) -в), перечисленных вьше. В каждом из этих случаев несложно получить противоречие.

Пусть теперь $v(G / Z)=4$. В этом случае если $A / Z$ - централизатор из $G / Z$ и $w(A / Z)=4$, то $Z(A)=Z \leqslant A^{\prime}$, и если $A / Z=C_{G / Z}(a Z)$, то $Z=\langle[a, b]\rangle$ для некоторого $b \in A$, т.е. $z$ врастает в силовскую $|z|$-подгруппу из $G / Z$.

Предположим сначала, что $F / Z$ абелева, т.е. $G / Z$ удовлетворяет условию теоремы 2.1 из [3]. Рассмотрим все двенадшать случаев из заключения этой теоремы.

1 ) Так как $z$ не может врастать в $Q_{8}$, то $z$ врастает в $F$ и $w(K) \leqslant 3$. Поэтому либо $K=Q_{8}$, либо $K=\langle a\rangle \lambda\langle b\rangle, a^{p_{1}}=b^{q_{1}^{2}}=1$. В силу $Z(F(G))=Z F(G)$ может быть только центральным склеиванием двух неабелевых подгрупп порядка $p^{3}$, т.е. $G$-группа типа 8.

2) Пусть $G / Z=\bar{G}_{1} \times \bar{G}_{2}$. Так как $w\left(C_{G / Z}(\bar{a})\right)=4$ для любого $\bar{a} \in \bar{G}_{1} \backslash 1$, то $|Z|$ должен делиться как на $p_{1}$ так и на $p_{2}$, что невозможно. 
3) В групе $\langle c\rangle$ найдется элемент $c_{0}$ простого порядка со свойством $w\left(C\left(c_{0}\right)\right)=4$. Поэтому $z$ врастает в силовскую $\left|c_{0}\right|$-подгруппу. Но тогда можно считать, что $\left|c_{0}\right|=p$ и $z$ врастает в $\left\langle b_{1}\right\rangle \times\left\langle c_{0}\right\rangle$. Так как $b_{1} \in C\left(c_{0}\right)$, то $\left\langle b_{1}\right\rangle$ является $c$-допустимой, т.е. $b_{1}^{c}=b_{1}^{\alpha}$, $\alpha \not \equiv 1(p)$. Но тогда в группе $G$ будем иметь $z=z^{c}=\left[b_{1}^{\alpha}, c_{0}\right]=z^{\alpha}$, что невозможно.

4) Пусть $b$-элемент простого порядка из $F$, для которого $C(b) \cap\langle c\rangle=\left\langle c_{0}\right\rangle \neq 1$. Тогда $|z|=|b|$. Если $z$ врастает в $\langle b\rangle \times\left\langle c_{0}\right\rangle$, то повторяем рассуждения из предыдущего абзаца. А если $z$ врастает в $\langle b\rangle \times\left\langle b_{1}\right\rangle$, то к противоречию приводит равенство $z=\left[b, b_{1}^{c_{0}}\right]$.

5) Так как в этом случае $w\left(C\left(a_{2}^{r}\right)\right)=4$, то $z$ врастает в подгруппу $\left\langle a_{1}\right\rangle \times\left\langle a_{2}\right\rangle$ и мы имеем группу типа 9 .

$6)$ В силу $w\left(C\left(a_{2}\right)\right)=4$ можно считать, что $z=\left[a_{1}, a_{2}^{r}\right]$. Но тогда в группе $G$ вьполняется неравенство $w\left(C\left(a_{2}^{q}\right)\right)>4$.

7) Случай $w(|a|)=4$ невозможен. Если же $w(|a|)=3$, то $|a|=2 n, z$ врастает в $\left\langle a^{n}\right\rangle \times\langle\tau\rangle$ и мы получаем группу типа 10 .

$8)$ Если $\tau$ - центральная инволюция из $S L(2,3)$, то $|C(\tau)|=24$. Поэтому $z$ должен врастать в силовскую 2 -подгруппу из $S L(2,3)$, что невозможно, так как $S_{2}(S L(2,3)) \cong$ Q8.

9) Так как $w\left(C\left(x^{q}\right)\right)=w(C(b))=4$ для некоторого $b \in\left(\left\langle b_{1}\right\rangle \times\left\langle b_{2}\right\rangle\right) \backslash 1$, то $|z|$ должен быть равен и $p$ и $q$, что противоречит тому, что $p \neq q$.

10) Из $w(C(c))=4$ следует, что $|z|=q$. Так как $p \neq q$, то должны вьполняться равенства $r=q$ и $z=[c, x]$. Действуя на последнее равенство элементом $y_{0} \in(C(x) \cap\langle y\rangle) \backslash 1$, снова получим противоречие.

11) Из $w(C(x))=4=w\left(C\left(b_{1} b_{2}\right)\right)$ получим $p=|z|=q$.

12) Так как $w\left(C\left(b_{1}\right)\right)=4$, то можно считать, что $z=\left[b_{1}, b_{2}\right]$. Действуя на это равенство элементом $y$, снова получим противоречие.

Предположим теперь, что группа $F / Z$ неабелева. Тогда $G / Z$ является группой из теоремы 2.2 из [3]. Рассмотрим эти групшы.

1) Так как $z$ может врасти только в нециклическую группу, то $|F|=p^{4}$ и $w(|c|) \leqslant 3$. Из того, что $v(F)=4$ и $F$ не является ни $m$-группой, ни центральньм склеиванием двух неабелевых групп порядка $p^{3}$, следует, что $G$ изоклинна одной из групп $G_{1}, G_{2}, G_{3}$ из теоремы 2.3. В случае групшы $G_{1}$ подгруппа $Z_{2}=\left\langle b_{1}^{p}\right\rangle \times\left\langle b_{2}^{p}\right\rangle c$-допустима. Но тогда она содержит $c$-допустимую циклическую подгруппу $\langle b\rangle$ порядка $p^{2}$, что невозможно, так как $b^{p}=z$, a $b^{c} \neq b$. Таким образом, в этом случае $G$ - группа типа 11 .

2) Здесь $p=q$ и из строения $F / Z$ следует, что $F$ может быть только группой типа $G_{2}$ из теоремы 2.3. Но тогда в обозначениях этой теоремы должны выполняться равенства $a_{2}^{c}=a_{2}^{\beta}, b_{1}^{c}=b_{1}$, откуда $z^{c}=z^{\beta}$, что невозможно.

В случае 3 ) тоже должно вьполняться равенство $p=q$. Так как для групп 3$)-7$ ) подгруппа $F$ не изоморфна $G_{i} / Z$ ни для какой групшы $G_{i}$ из теоремы 2.3 , то эти случаи невозможны.

8) $F(G)$ может быть только группой типа $G_{3}$ из теоремы 2.3. Пусть $G=F \lambda\langle d\rangle$. Тогда в обозначениях теоремы 2.3 можно считать, что $a^{d}=a^{\alpha}$ и $b^{d}=b^{\alpha}$. Из $[a, b]=z$ получим $\alpha^{2}=1$, что противоречит условию $w(|d|)=2$.

9) В этом случае должны выполняться условия $|z|=p$ и $w(|c|)<3$. Но тогда $F=F(G)$ групша порядка $p^{4}$ с $|Z(F)|=p$, т.е. $F$ одна из груп, перечисленных перед теоремой. В случае а) мы имеем $b^{c} \equiv b^{\alpha \beta}, a^{c} \equiv a^{\alpha}, x^{c} \equiv x^{\beta}(\bmod Z)$. Так как $z=a^{p}$, то $\alpha=1$. Но тогда из $[b, x]=z$ получим $z=\left[b^{\beta}, x^{\beta}\right]=z^{\beta^{2}}$, т.е. $\beta^{2}=1$, что противоречит тому, что $\wp(\beta)=|c|$ и $w(|c|)=2$. 
В случае б) имеем $a^{c} \equiv a^{\alpha} \beta, b^{c} \equiv b^{\alpha}, x^{c} \equiv x^{\beta}(\bmod Z)$. Отсюда $z=[a, x]^{c}=z^{\alpha \beta^{2}}$, т.е. $\alpha \beta^{2}=1$. Но тогда $\alpha \beta=\beta^{-1}$ и, следовательно, $\wp(\alpha \beta)=\wp(\beta)$. Но тогда с учетом того, что $w(|c|)<3$, в пункте 9 теоремы 2.2 из [3] может вьполняться только случай а). Из $\alpha=1$ следует, что $\beta^{2}=1$, что невозможно.

Случай в) аналогично приводится к противоречию.

10) Группа $F$ порядка $p^{4}$ с центром порядка $p$ не может иметь такую фактор-группу по центру (см. вьше).

11) Снова используем строение груп, перечисленных перед теоремой. В случаях а) и в) из $a^{d}=a^{s}, z=a^{p}$ и $z^{d}=z$ сразу получаем противоречие. А в случае б) противоречие следует из того, что $\langle c\rangle$ должна действовать на $F / Z_{2}(F)$ неприводимо, а подгруппа $\langle b\rangle Z_{2}(F)=C_{F}\left(Z_{2}(F)\right)$ инвариантна.

\section{СПИСОК ЦИТИРОВАННОЙ ЛИТЕРАТУРЫ}

[1] Bianchi M., Manz O. Groups with small centralizers of non-central elements // Boll. Un. Mat. Ital. Ser. A. 1990. V. 4. № 7. P. 365-370.

[2] Scarselli A. Gruppi con piccoli centralizzanti // Boll. Un. Mat. Ital. 1992. V. 6-B. № 7. P. 649-663.

[3] Антонов В. А., Тюрина И. А., Ческидов А. П. Группы с малыми централизаторами // Матем. заметки. 2001. Т. 69. № 5. С. 643-655.

[4] Schmidt R. Zentralisatorverbände endlicher Gruppen // Rend. Sem. Math. Univ. Padova. 1970. № 44. P. 97-131.

[5] Hall Ph. The classification of prime-power groups // J. Reine Angew. Math. 1940. V. 182. P. $130-141$.

[6] Rocke D. M. p-groups with abelian centralizers // Proc. London Math. Soc. (3). 1975. V. 30. № 1. P. $55-75$.

[7] Антонов В. А. Конечные группы с модулярной решеткой централизаторов // Алгебра и логика. 1987. Т. 26. №6. С. 653-683.

[8] Verardi L. On groups whose noncentral elements have the same finite number of conjugates // Boll. Un. Mat. Ital. Ser. A. 1988. V. 3. P. 391-400.

[9] Huppert B. Endliche Gruppen, 1. Berlin-Heidelberg-New York: Springer-Verlag, 1967.

[10] Гаген Т. М. К теории конечных групп. М.: Мир, 1979.

(В.А. Антонов, И. А. Тюрина) Южно-уральский государственный университет 